\title{
Invitation to operadic dynamics
}

Eugen $P A A L$

Department of Mathematics, Tallinn University of Technology, Ehitajate tee 5, 19086 Tallinn, Estonia

E-mail: eugen.paal@ttu.ee

\begin{abstract}
Based on the Gerstenhaber Theory, clarification is made of how operadic dynamics may be introduced. Operadic observables satisfy the Gerstenhaber algebra identities and their time evolution is governed by operadic evolution equation. The notion of an operadic Lax pair is also introduced. As an example, an operadic (representation of) harmonic oscillator is proposed.
\end{abstract}

2000 MSC: 18D50, 70G60

\section{Introduction and outline of the paper}

In 1963, Gerstenhaber invented [1] an operad calculus in the Hochschild complex of an associative algebra; operads were introduced under the name of pre-Lie systems. In the same year, Stasheff constructed [13] (see also [11]) quite an original geometrical operad, which nowadays is called an associahedra. The notion of an operad was further formalised by May [10] as a tool for iterated loop spaces. The main principles of the operad calculus (brace algebra) were presented by Gerstenhaber and Voronov [3, 14]. Some quite remarkable research activity in the operad theory and its applications can be observed in the last decade (e.g $[8,12,9])$. It may be said that operads are also becoming an important tool for Quantum Field Theory and deformation quantization [6].

Today, much attention is given to static operadic constructions. For dynamical operations one has to prescribe their time evolution. In this paper, based on the Gerstenhaber Theory, clarification is given on how operadic dynamics may be introduced.

We start from simple algebraic axioms. Basic algebraic constructions associated with linear operads are introduced. Their properties and the first derivation deviations for the coboundary operator are presented explicitly. Under certain conditions (a formal associativity constraint), the Gerstenhaber algebra structure appears in the associated cohomology of an operad.

The operadic dynamics may be introduced by simple and natural analogy with the Hamiltonian version. Operadic observables satisfy the Gerstenhaber algebra identities and their time evolution is governed by the operadic analogue of the Hamiltonian equations, the operadic evolution equation. The latter describes the time evolution of operations. In particular, the notion of an operadic Lax pair may be introduced as well. As an example, an operadic (representation of) harmonic oscillator is proposed.

\section{Operad}

Let $K$ be a unital associative commutative ring, and let $C^{n}(n \in \mathbb{N})$ be unital $K$-modules. For homogeneous $f \in C^{n}$, we refer to $n$ as the degree of $f$ and often write (when it does not cause confusion) $f$ instead of $\operatorname{deg} f$. For example, $(-1)^{f} \doteq(-1)^{n}, C^{f} \doteq C^{n}$ and $\circ_{f} \doteq \circ_{n}$. Also, it is convenient to use the reduced degree $|f| \doteq n-1$. Throughout this paper, we assume that $\otimes \doteq \otimes_{K}$. 
Definition 2.1 (operad). A linear (nonsymmetric) operad with coefficients in $K$ is a sequence $C \doteq\left\{C^{n}\right\}_{n \in \mathbb{N}}$ of unital $K$-modules (an $\mathbb{N}$-graded $K$-module), such that the following conditions are held to be true.

(1) For $0 \leq i \leq m-1$ there exist partial compositions

$$
\circ_{i} \in \operatorname{Hom}\left(C^{m} \otimes C^{n}, C^{m+n-1}\right), \quad\left|\circ_{i}\right|=0
$$

(2) For all $h \otimes f \otimes g \in C^{h} \otimes C^{f} \otimes C^{g}$, the composition (associativity) relations hold,

$$
\left(h \circ_{i} f\right) \circ_{j} g= \begin{cases}(-1)^{|f||g|}\left(h \circ_{j} g\right) \circ_{i+|g|} f & \text { if } 0 \leq j \leq i-1, \\ h \circ_{i}\left(f \circ_{j-i} g\right) & \text { if } i \leq j \leq i+|f| \\ (-1)^{|f||g|}\left(h \circ_{j-|f|} g\right) \circ_{i} f & \text { if } i+f \leq j \leq|h|+|f| .\end{cases}
$$

(3) Unit $I \in C^{1}$ exists such that

$$
\mathrm{I} \circ_{0} f=f=f \circ_{i} \mathrm{I}, \quad 0 \leq i \leq|f|
$$

In the second item, the first and third parts of the defining relations turn out to be equivalent.

Example 2.2 (endomorphism operad [1]). Let $L$ be a unital $K$-module and $\mathcal{E}_{L}^{n} \doteq \mathcal{E} n d_{L}^{n} \doteq$ $\operatorname{Hom}\left(L^{\otimes n}, L\right)$. Define the partial compositions for $f \otimes g \in \mathcal{E}_{L}^{f} \otimes \mathcal{E}_{L}^{g}$ as

$$
f \circ_{i} g \doteq(-1)^{i|g|} f \circ\left(\mathrm{id}_{L}^{\otimes i} \otimes g \otimes \mathrm{id}_{L}^{\otimes(|f|-i)}\right), \quad 0 \leq i \leq|f|
$$

Then $\mathcal{E}_{L} \doteq\left\{\mathcal{E}_{L}^{n}\right\}_{n \in \mathbb{N}}$ is an operad (with the unit $\mathrm{id}_{L} \in \mathcal{E}_{L}^{1}$ ) called the endomorphism operad of $L$.

Therefore, algebraic operations can be seen as elements of an endomorphism operad.

Example 2.3 (coendomorphism operad). Let $L$ be a $K$-space and

$$
\overline{\mathcal{E}}_{L}^{n} \doteq \mathcal{C} o E n d_{L}^{n} \doteq \operatorname{Hom}\left(L, L^{\otimes n}\right)
$$

Define the partial compositions for $f \otimes g \in \overline{\mathcal{E}}_{L}^{f} \otimes \overline{\mathcal{E}}_{L}^{g}$ as

$$
f \circ_{i} g \doteq(-1)^{i|g|}\left(\mathrm{id}_{L}^{\otimes i} \otimes g \otimes \mathrm{id}_{L}^{\otimes(|f|-i)}\right) \circ f, \quad 0 \leq i \leq|f|
$$

Then $\overline{\mathcal{E}}_{L} \doteq\left\{\overline{\mathcal{E}}_{L}^{n}\right\}_{n \in \mathbb{N}}$ is an operad (with the unit $\operatorname{id}_{L} \in \overline{\mathcal{E}}_{L}^{1}$ ) called the coendomorphism operad of $L$.

Therefore, algebraic co-operations can be seen as elements of a coendomorphism operad.

Just as elements of a vector space are called vectors, it is natural to call elements of an abstract operad operations.

\section{Cup and braces}

Throughout this paper, fix a binary operation $\mu \in C^{2}$ in an operad $C$.

Definition 3.1. The cup-multiplication $\smile: C^{f} \otimes C^{g} \rightarrow C^{f+g}$ is defined by

$$
f \smile g \doteq(-1)^{f}\left(\mu \circ_{0} f\right) \circ_{f} g \in C^{f+g}, \quad|\smile|=1
$$

The pair $\operatorname{Cup} C \doteq\{C, \smile\}$ is called a $\smile$-algebra (cup-algebra) of $C$. 
Example 3.2. For the endomorphism operad (Example 2.2) $\mathcal{E}_{L}$ one has

$$
f \smile g=(-1)^{f g} \mu \circ(f \otimes g), \quad \mu \otimes f \otimes g \in \mathcal{E}_{L}^{2} \otimes \mathcal{E}_{L}^{f} \otimes \mathcal{E}_{L}^{g}
$$

Definition 3.3. The total composition $\bullet: C^{f} \otimes C^{g} \rightarrow C^{f+|g|}$ is defined by

$$
f \bullet g \doteq \sum_{i=0}^{|f|} f \circ_{i} g \in C^{f+|g|}, \quad|\bullet|=0
$$

The pair Com $C \doteq\{C, \bullet\}$ is called the composition algebra of $C$.

Definition 3.4 (tribraces). Define the Gerstenhaber tribraces $\{\cdot, \cdot, \cdot\}$ as a double sum

$$
\{h, f, g\} \doteq \sum_{i=0}^{|h|-1} \sum_{i+f}^{|f|+|h|}\left(h \circ_{i} f\right) \circ_{j} g \in C^{h+|f|+|g|}, \quad|\{\cdot, \cdot, \cdot\}|=0
$$

Definition 3.5 (tetrabraces). The tetrabraces $\{\cdot, \cdot, \cdot, \cdot\}$ are defined by

$$
\{h, f, g, b\} \doteq \sum_{i=0}^{|h|-2} \sum_{j=i+f}^{|h|+|f|-1} \sum_{k=j+g}^{|h|+|f|+|g|}\left(\left(h \circ_{i} f\right) \circ_{j} g\right) \circ_{k} b \in C^{h+|f|+|g|+|b|}, \quad|\{\cdot, \cdot, \cdot, \cdot\}|=0
$$

It turns out that

$$
f \smile g=(-1)^{f}\{\mu, f, g\}
$$

In general, $\operatorname{Cup} C$ is a non-associative algebra. By denoting $\mu^{2} \doteq \mu \bullet \mu$, it turns out that the associator in $\operatorname{Cup} C$ reads

$$
(f \smile g) \smile h-f \smile(g \smile h)=\left\{\mu^{2}, f, g, h\right\}
$$

Therefore the formal associator (micro-associator) $\mu^{2}$ is an obstruction to the associativity of $\operatorname{Cup} C$. For an endomorphism operad $\mathcal{E}_{L}$, the ternary operation $\mu^{2}$ also reads as an associator:

$$
\mu^{2}=\mu \circ\left(\mu \otimes \operatorname{id}_{L}-\operatorname{id}_{L} \otimes \mu\right), \quad \mu \in \mathcal{E}_{L}^{2}
$$

\section{Gerstenhaber brackets}

In an operad $C$, the Getzler identity

$$
(h, f, g) \doteq(h \bullet f) \bullet g-h \bullet(f \bullet g)=\{h, f, g\}+(-1)^{|f||g|}\{h, g, f\}
$$

holds, which easily implies the Gerstenhaber identity

$$
(h, f, g)=(-1)^{|f||g|}(h, g, f)
$$

The Gerstenhaber brackets $[\cdot, \cdot]$ are defined in Com $C$ as a graded commutator by

$$
[f, g] \doteq f \bullet g-(-1)^{|f||g|} g \bullet f=-(-1)^{|f||g|}[g, f], \quad|[\cdot, \cdot]|=0
$$

The commutator algebra of $\operatorname{Com} C$ is denoted as $\operatorname{Com}^{-} C \doteq\{C,[\cdot, \cdot]\}$. By using the Gerstenhaber identity, one can prove that $\mathrm{Com}^{-} C$ is a graded Lie algebra. The Jacobi identity reads

$$
(-1)^{|f||h|}[[f, g], h]+(-1)^{|g||f|}[[g, h], f]+(-1)^{|h||g|}[[h, f], g]=0
$$




\section{Coboundary operator}

In an operad $C$, by using the Gerstenhaber brackets, a (pre-)coboundary operator $\partial \doteq \partial_{\mu}$ may be defined by

$$
\begin{aligned}
\partial f & \doteq \operatorname{ad}_{\mu}^{\text {right }} f \doteq[f, \mu] \doteq f \bullet \mu-(-1)^{|f|} \mu \bullet f \\
& =f \smile \mathrm{I}+f \bullet \mu+(-1)^{|f|} \mathrm{I} \smile f, \quad \operatorname{deg} \partial=+1=|\partial|
\end{aligned}
$$

It follows from the Jacobi identity in $\mathrm{Com}^{-} C$ that $\partial$ is a (right) derivation of $\mathrm{Com}^{-} C$,

$$
\partial[f, g]=(-1)^{|g|}[\partial f, g]+[f, \partial g]
$$

and one has the commutation relation

$$
\left[\partial_{f}, \partial_{g}\right] \doteq \partial_{f} \partial_{g}-(-1)^{|f||g|} \partial_{g} \partial_{f}=\partial_{[g, f]}
$$

Therefore, since $|\mu|=+1$ is odd, then

$$
\partial_{\mu}^{2}=\frac{1}{2}\left[\partial_{\mu}, \partial_{\mu}\right]=\frac{1}{2} \partial_{[\mu, \mu]}=\partial_{\mu \bullet \mu}=\partial_{\mu^{2}}
$$

Here we assumed that $2 \neq 0$, the proof for an arbitrary characteristic may be found from [5]. But $\partial$ need not be a derivation of $\operatorname{Cup} C$, and $\mu^{2}$ again appears as an obstruction:

$$
\partial(f \smile g)-f \smile \partial g-(-1)^{g} \partial f \smile g=(-1)^{g}\left\{\mu^{2}, f, g\right\}
$$

\section{Derivation deviations}

The derivation deviation of $\partial$ over $\bullet$ is defined by

$$
(\operatorname{dev} \bullet)(f \otimes g) \doteq \partial(f \bullet g)-f \bullet \partial g-(-1)^{|g|} \partial f \bullet g
$$

Theorem 6.1. In a pre-operad $C$, one has

$$
(-1)^{g}\left(\operatorname{dev}_{\bullet} \partial\right)(f \otimes g)=f \smile g-(-1)^{f g} g \smile f
$$

Proof. The full proof is presented in [4].

The derivation deviation of $\partial$ over $\{\cdot, \cdot, \cdot\}$ is defined by

$$
\begin{aligned}
\left(\operatorname{dev}_{\{\cdot, \cdot, \cdot\}} \partial\right)(h \otimes f \otimes g) \doteq \partial\{h, f, g\} & -\{h, f, \partial g\} \\
& -(-1)^{|g|}\{h, \partial f, g\}-(-1)^{|g|+|f|}\{\partial h, f, g\}
\end{aligned}
$$

Theorem 6.2. In a pre-operad $C$, one has

$$
(-1)^{g}\left(\operatorname{dev}_{\{\cdot, \cdot, \cdot\}} \partial\right)(h \otimes f \otimes g)=(h \bullet f) \smile g+(-1)^{|h| f} f \smile(h \bullet g)-h \bullet(f \smile g)
$$

Proof. The full proof is presented in [5].

Therefore the left translations in $\operatorname{Com} C$ are not derivations of $\operatorname{Cup} C$, the corresponding deviations are related to $\operatorname{dev}_{\{,,,,\}} \partial$. It turns out that the right translations in $\operatorname{Com} C$ are derivations of $\operatorname{Cup} C$,

$$
(f \smile g) \bullet h=f \smile(g \bullet h)+(-1)^{|h| g}(f \bullet h) \smile g
$$

By combining this formula with the one from Theorem 6.2 we obtain

Theorem 6.3. In a pre-operad $C$, one has

$$
(-1)^{g}\left(\operatorname{dev}_{\{\cdot,,, \cdot\}} \partial\right)(h \otimes f \otimes g)=[h, f] \smile g+(-1)^{|h| f} f \smile[h, g]-[h, f \smile g]
$$




\section{Gerstenhaber Theory}

Now, clarification can be supplied to show how the Gerstenhaber algebra can be associated with a linear operad. If (formal associativity) $\mu^{2}=0$ holds, then $\partial^{2}=0$, which in turn implies $\operatorname{Im} \partial \subseteq$ Ker $\partial$. Then one can form an associated cohomology (N-graded module) $H(C) \doteq \operatorname{Ker} \partial / \operatorname{Im} \partial$ with homogeneous components

$$
H^{n}(C) \doteq \operatorname{Ker}\left(C^{n} \stackrel{\partial}{\rightarrow} C^{n+1}\right) / \operatorname{Im}\left(C^{n-1} \stackrel{\partial}{\rightarrow} C^{n}\right)
$$

where, by convention, $\operatorname{Im}\left(C^{-1} \stackrel{\partial}{\rightarrow} C^{0}\right) \doteq 0$. Also, in this $\left(\mu^{2}=0\right)$ case, $\operatorname{Cup} C$ is associative,

$$
(f \smile g) \smile h=f \smile(g \smile h)
$$

and $\partial$ is a derivation of $\operatorname{Cup} C$. Remember from previously that $\mathrm{Com}^{-} C$ is a graded Lie algebra and $\partial$ is a derivation of $\mathrm{Com}^{-} C$. Due to the derivation properties of $\partial$, the multiplications $[\cdot, \cdot]$ and $\smile$ induce corresponding (factor) multiplications on $H(C)$, which we denote by the same symbols. Then $\{H(C),[\cdot, \cdot]\}$ is a graded Lie algebra. It follows from Theorem 6.1 that the induced $\smile$-multiplication on $H(C)$ is graded commutative,

$$
f \smile g=(-1)^{f g} g \smile f
$$

for all $f \otimes g \in H^{f}(C) \otimes H^{g}(C)$, hence $\{H(C), \smile\}$ is an associative graded commutative algebra. It follows from Theorem 6.3 that the graded Leibniz rule holds,

$$
[h, f \smile g]=[h, f] \smile g+(-1)^{|h| f} f \smile[h, g]
$$

for all $h \otimes f \otimes g \in H^{h}(C) \otimes H^{f}(C) \otimes H^{g}(C)$. At last, it is also relevant to note that

$$
0=|[\cdot, \cdot]| \neq|\smile|=1
$$

In this way, the triple $\{H(C), \smile,[\cdot, \cdot]\}$ turns out to be a Gerstenhaber algebra [2]. The defining relations of a Gerstenhaber algebra are (G1)-(G6).

In the case of an endomorphism operad, the Gerstenhaber algebra structure appears on the Hochschild cohomology of an associative algebra [1]. This is the essence of the Gerstenhaber Theory.

In particular, in the case of a coendomorphism operad, the Gerstenhaber algebra structure appears on the Cartier cohomology of a coassociative coalgebra.

\section{Operadic dynamics}

Assume that $K \doteq \mathbb{R}$ or $K \doteq \mathbb{C}$. It is known that the Poisson algebras can be seen as an algebraic abstraction of mechanics. Consider the following figurative commutative diagram:

$$
\begin{array}{ccc}
\text { Poisson algebras } & \stackrel{\text { algebra }}{\longleftarrow} \text { mechanics } \\
\text { ? }
\end{array}
$$

Gerstenhaber algebras $\stackrel{\text { algebra }}{\longleftarrow}$ operadic mechanics

Concisely speaking, operadic observables are elements of a Gerstenhaber algebra. The time evolution of an operadic operadic obervable $f$ is governed by the operadic evolution equation

$$
\frac{d f}{d t}=[H, f] \doteq H \bullet f-(-1)^{|H||f|} f \bullet H
$$


with the (model-dependent) operadic Hamiltonian $H$. The most simple assumption for its degree is

$$
\left|\frac{d}{d t}\right|=|H|=0 \quad \Longrightarrow \quad[H, f] \doteq H \bullet f-f \bullet H
$$

In particular,

$$
|H|=|f|=0 \quad \Longrightarrow \quad[H, f]=H \circ f-f \circ H
$$

and in this case one finds the well-known evolution equation

$$
\frac{d f}{d t}=[H, f] \doteq H \circ f-f \circ H
$$

In this way one can describe the time evolution of operations. In particular, one can propose the

Definition 8.1 (operadic Lax pair). Allow a classical dynamical system to be described by the evolution equations

$$
\frac{d x_{i}}{d t}=f_{i}\left(x_{1}, \ldots, x_{n}\right), \quad i=1, \ldots, n
$$

An operadic Lax pair is a pair of homogeneous operations $L, M \in C$, such that the above system of evolution equations is equivalent to the operadic Lax equation

$$
\frac{d L}{d t}=[M, L] \doteq M \bullet L-(-1)^{|M||L|} L \bullet M
$$

Evidently, $|M|=0$ is the most simple assumption and the degree constraints $|M|=|L|=0$ give rise to ordinary Lax pair [7].

Endomorphism and co-endomorphism operads are the most natural objects for modelling operadic dynamical systems.

Surprisingly, examples are at hand. By using the Lax pairs one may extend these to operadic area via the operadic Lax equation.

Example 8.2 (operadic harmonic oscillator). Consider the classical Lax pair for the harmonic oscillator:

$$
L_{c l}=\left(\begin{array}{cc}
p & \omega q \\
\omega q & -p
\end{array}\right), \quad M=\left(\begin{array}{cc}
0 & -\omega / 2 \\
\omega / 2 & 0
\end{array}\right)
$$

Since the Hamiltonian is

$$
H(q, p)=\frac{1}{2}\left(p^{2}+\omega^{2} q^{2}\right)
$$

one can use the Hamiltonian canonical equations

$$
\frac{d q}{d t}=\frac{\partial H}{\partial p}=p, \quad \frac{d p}{d t}=-\frac{\partial H}{\partial q}=-\omega^{2} q
$$

to obtain

$$
\frac{d L}{d t}=\frac{\partial L}{\partial q} \frac{d q}{d t}+\frac{\partial L}{\partial p} \frac{d p}{d t}=p \frac{\partial L}{\partial q}-\omega^{2} q \frac{\partial L}{\partial p}
$$

Therefore the linear partial differential equation for the operadic variable $L(q, p)$ reads

$$
p \frac{\partial L}{\partial q}-\omega^{2} q \frac{\partial L}{\partial p}=M \bullet L-L \bullet M
$$

By integrating one gains a sequence of operations called an operadic (representation of) harmonic oscillator. 


\section{Acknowledgement}

Research was in part supported by the Estonian Science Foundation, Grants 5634 and 6912 . The author is grateful to Piret Kuusk for reading the preliminary manuscript, and for contributing valuable remarks and discussions.

\section{References}

[1] M. Gerstenhaber. The cohomology structure of an associative ring. Ann. of Math. 78 (1963), 267-288.

[2] M. Gerstenhaber, A. Giaquinto, and S. D. Schack. Algebras, bialgebras, quantum groups, and algebraic deformations. Contemp. Math. 134 (1992), 51-92.

[3] M. Gerstenhaber and A. A. Voronov. Homotopy G-algebras and moduli space operad. Intern. Math. Res. Notices, 1995, No. 3, 141-153.

[4] L. Kluge and E. Paal. On derivation deviations in an abstract pre-operad. Comm. Algebra, 29 (2001), 1609-1626.

[5] L. Kluge, E. Paal, and J. Stasheff. Invitation to composition. Comm. Algebra, 28 (2000), 1405-1422.

[6] M. Kontsevich. Operads and motives in deformation quantization. Lett. Math. Phys. 48 (1999), 35-72.

[7] P. D. Lax. Integrals of nonlinear equations of evolution and solitary waves. Comm. Pure Applied Math. 21 (1968), 467-490.

[8] J.-L. Loday, J. Stasheff, and A. A. Voronov, Eds. Operads: Proceedings of Renaissance Conferences. Contemp. Math. 202 (1997).

[9] M. Markl, S. Shnider, and J. Stasheff. Operads in Algebra, Topology and Physics. AMS, 2002.

[10] J. P. May. The Geometry of Iterated Loop Spaces. Lecture Notes in Math. 271, 1972.

[11] S. Shnider and S. Sternberg. Quantum Groups from Coalgebras to Drinfeld Algebras. Boston, Int. Press, 1993.

[12] V. A. Smirnov. Simplicial and Operad Methods in Algebraic Topology. AMS, Transl. Math. Monogr. 198, 2001.

[13] J. D. Stasheff. Homotopy associativity of H-spaces, I, II. Trans. Amer. Math. Soc. 108 (1963), 275-292, 293-312.

[14] A. A. Voronov and M. Gerstenhaber. Higher-order operations on the Hochschild complex. Funktsional. Anal. i Prilozhen. 29 (1995), 1-6 (in Russian). 